\title{
A Move Towards Focused Parathyroidectomy in BSMMU Even Under Local Anesthesia
}

\author{
Jawhar Lal Singha', Saifuddin Ahmed², AHM Towhidul Alam, Nasim Sobhan Khondokar ${ }^{4}$, \\ Syed Serajul Karim ${ }^{5}$ \\ ${ }^{1}$ MS Student, ${ }^{2}$ Associate Professor, ${ }^{3}$ Associate Professor, ${ }^{5}$ Professor \& Chairman, Department of Surgey, Bangabandhu Sheikh Mujib Medical \\ University (BSMMU), Dhaka, Bangladesh, ${ }^{4}$ Associate Professor, Department of Surgery, Shahid Ziaur Rahman Medial College, Bogra.
}

\begin{abstract}
:
Parathyroidectomy needs skill and experience for its tiny size and variation in number and location. The retrospective quasi-experimental study of 26 parathyroid operations was done in endocrine surgery unit of BSMMU during October 1994 to March 2007. All patients were within 31 to 50 years of age with female preponderance (M:F=1:5.5). All the patients had primary hyperparathyroidism diagnosed biochemically with variable rise of serum calcium and serum intact parathormone level and finally proved as single parathyroid adenoma after surgery. Different preoperative localization tests were done in 22 patients with positive impression in all except 2(7.69\%) sestamibi scan. The sestamibi scan showed 50\% (2/4) negative impression which, later on, was proved as 100\% false negative at surgery. Bilateral neck exploration (BNE) was done on 18 patients with $94.44 \%$ success of surgery and 'focused' parathyroidectomy by unilateral neck exploration (UNE) was done on 7 patients of whom 4 underwent local anaesthesia with 100\% success of gland removal. Temporary postoperative hypocalcaemia was observed in 22 patients. Full recovery from hypocalcaemia was in 20(90.90\%) patients though 2 (7.69\%) patients with late diagnosis showed irreversible outcome not due to surgery but due to postoperative complex renal and cardiovascular deterioration. Focused parathyroidectomy can be safely done even under local anaesthesia in tertiary hospital.
\end{abstract}

Key words: Primary hyperparathyroidism, preoperative localization, focused parathyroidectomy, local anaesthesia.

[BSMMU J 2009; 2(1): 31-35]

\section{Introduction:}

Primary hyperparathyroidism is usually a late diagnosis as a cause of hypercalcaemia. Diagnosis may be symptomatic or incidental. Most often endocrine surgeon is demanded for surgical management of hyperactive parathyroid gland(s) after diagnosis by specialist urologist, orthopaedic surgeon, endocrinologist or even psychiatrist. After biochemical diagnosis by raised serum calcium and serum parathormone (PTH), preoperative localization tests are done to identify the number and location of hyperactive glands for appropriate approach to gland removal. Parathyroid surgery is one of the highly technical procedures due to its tiny size, variation of location and infrequent and limited exposure and experience of the surgeon. In the past decades, due to reduced sensitivity and specificity of localization tests, localization of a 'skilled parathyroid surgeon' was preferred to tests and parathyroidectomy by bilateral neck exploration (BNE) was the standard modality of treatment. But in recent years,

Correspondence to : Dr. Saifuddin Ahmed, Associate Professor, Department of Surgery, BSMMU, Dhaka-1000, E-mail address: saifuddin1962@live.com due to the grace of sophisticated imaging, localization of the gland(s) is more accurately possible ${ }^{1-6}$ and focused parathyroidectomy by unilateral neck exploration (UNE) is welcomed with similar success rate. ${ }^{7-9}$ The rationale behind focused parathyroidectomy is the incidence of single gland disease, which is $85-90 \% .^{1,7,8}$ Shorter operative time, less postoperative morbidity, less hospital stay and easier re-operation if recurrence develops are added advantages. Instead of general anaesthesia local anaesthesia can be used in well-localized gland and can be used confidently if the gland is clinically palpable. ${ }^{1,10}$ Different endoscopic, radio- and video-assisted surgeries along with peroperative gamma-probe detection for presumed multigland disease are also being done in developed centers. ${ }^{11,12}$ Intraoperative hormone measurement (50\% reduction of pre-excision baseline within 15 minutes of surgery, called 'biochemical frozen section') predicts gland removal and postoperative normocalcaemia though doubt exists about its necessity in recent investigators. ${ }^{12}$ Whatever the procedure of parathyroidectomy or anaesthesia it should be done without delay after diagnosis to reduce the risk of musculoskeletal, renal and cardiovascular complications. ${ }^{13}$ 


\section{Methods:}

The quasi-experimental study performed in endocrine surgery unit of BSMMU was based on retrospective collection and compilation of data of 26 parathyroid patients operated by single endocrine surgeon from October 1994 to March 2007. Randomization of sampling was not possible due to rarity of the pathology. The data was collected from hospital records, patient's documents and direct contact between patients and surgeon. Most of the patients were transferred in endocrine surgery unit from other specialties like urology, orthopedics, nephrology, endocrinology or psychiatry. A small number of patients came directly to surgeon. Before going to surgery investigations for diagnosis, localization, assessment of renal, musculoskeletal and cardiovascular function and general anaesthesia were done. For preoperative localization ultrasonography, scintigraphy (ThalliumTechnitium \& sestamibi scan) or CT scan were done in most patients. Local anaesthesia (field block with $2 \%$ lignocaine and $0.5 \%$ bupevacaine in equal ratio) was used in 4 cases with palpable parathyroids. Unilateral focused parathyroidectomy was done on 8 patients and other 18 underwent bilateral neck exploration. Success rate of gland removal between two groups was tested by chi-square formula as were the postoperative complications. Median follow-up was 6.5 years. 3 patients dropped out from endocrine surgical follow-up.

\section{Results:}

All the patients had hypercalcaemia of variable level at diagnosis. Females of 31-50 years (61.53\%) were most commonly affected $(\mathrm{M}: \mathrm{F}=1: 5.5)$. Bilateral nephrolithiasis was the commonest (42.30\%) mode of presentation. Sestamibi scan was negative in 50\%( 2/4) cases. But all were found as single gland disease at operation. All 8 patients undergoing UNE including those under local anaesthesia had 100\% successful parathyroidectomy. In BNE success rate was $94.44 \%$. Two patients were unfit for general anaesthesia with very high level of PTH (2500 $\& 1357 \mathrm{pgm} / \mathrm{ml})$. They were operated successfully under local anaesthesia but developed irreversible cardiovascular, renal and metabolic derangement. All others got recovery from transient hypocalcaemia.

Table-I

Age and sex distribution of patients $(n=26)$ :

\begin{tabular}{lcccc}
\hline Age & Female & Male & M: F & Total \\
& $\mathrm{N}(\%)=22$ & $\mathrm{~N}(\%)=4$ & & $\mathrm{~N}(\%)$ \\
\hline 15-30 yrs & $5(19.23)$ & 0 & $1: 5.5$ & $5(19.23)$ \\
31-50 yrs & $16(61.53)$ & $4(15.38)$ & & $20(76.92)$ \\
$>50$ yrs & $1(3.84)$ & 0 & & $1(3.84)$ \\
\hline
\end{tabular}

Table-II

Clinical presentation $(n=26)$ :

\begin{tabular}{lc}
\hline Type of presentation & Number (\%) \\
\hline Bone pain and cyst (single/multiple) & $\begin{array}{c}\text { 5(19.23) ; (Long } \\
\text { bone-4; Jaw-1) }\end{array}$ \\
Bone pain and fracture & $2(7.69)$ \\
Bilateral nephrolithiasis & $11(42.30)$ \\
Nephrocalcinosis & $3(11.53)$ \\
Recurrent renal stone & $4(15.38)$ \\
Pathological fracture with bilateral & $1(3.84)$ \\
renal stone & \\
\hline
\end{tabular}

Table-III

Biochemical diagnosis $(n=26)$ :

\begin{tabular}{lccc}
\hline Test & Level & No. of pts (\%) & Mean \\
\hline S. Calcium & $>11$ & $15(57.69)$ & \\
(8.5-10.5 mg/dl) & $10-11$ & $9(34.61)$ & 11.28 \\
& $9-10$ & $2(7.69)$ & \\
Blood PTH & $53-500$ & $3(11.53)$ & \\
(7-53 pgm/ml) & $500-1000$ & $8(30.76)$ & 947.1 \\
& $>1000$ & $15(57.69)$ & \\
24 h urine calcium & $<100$ & 0 & \\
(100-300 mg/24h) & $100-200$ & $16(61.53)$ & 188.46 \\
& $200-300$ & $10(38.46)$ & \\
S. creatinine & $>2$ & $4(15.38)$ & \\
(.6-1.3 mg/dl) & $1-2$ & $8(30.78)$ & 1.36 \\
& $<1$ & $149(53.84)$ & \\
S. Phosphate & $<3$ & $7(26.92)$ & 2.9 \\
(2.48-4.38 mg/dl) & $3-4$ & $5(19.23)$ & \\
& 4 & $(15.38) ;$ Not & \\
& \multicolumn{3}{c}{ done- 10(38.46) } \\
\hline
\end{tabular}

Table-IV

Preoperative localization $(n=22)$ :

\begin{tabular}{lcc}
\hline Way of localization & $\begin{array}{c}\text { +ve } \\
\mathrm{N}(\%)\end{array}$ & $\begin{array}{c}\text {-ve } \\
\mathrm{N}(\%)\end{array}$ \\
\hline Clinical detection (3) & $3(11.53)$ & \\
Ultrasonogram (5) & $5(19.23)$ & \\
Thallium-technitium subtraction & $9(34.61)$ & \\
scan (9) & & \\
Sestamibi scan (4) & $2(7.69)$ & $2(7.69)$ \\
CT scan (1) & $1(3.84)$ & \\
Not done (4) & - & - \\
\hline
\end{tabular}


Table-V

Parathyroidectomy $(n=26)$ done by :

\begin{tabular}{lccccc}
\hline & $\begin{array}{c}\text { Bilateral neck } \\
\text { exploration (BNE), } \\
\mathrm{n}=18\end{array}$ & $\begin{array}{c}\text { Unilateral neck } \\
\text { exploration (UNE), } \\
\mathrm{n}=8 \\
\text { (Focused-7 } \\
\text { Extended-1) }\end{array}$ & $\begin{array}{c}\text { Chi-square } \\
\text { value }\end{array}$ & df & P value \\
Success & $17(94.4 \%)$ & $8(100 \%)$ & .462 & 1 & $1.00^{*}$ \\
Failure & 1 & 0 & & & \\
\hline
\end{tabular}

* Fisher's Exact Test was done

Table-VI

Peroperative localization $(n=26)$ :

\begin{tabular}{lc}
\hline Location & Number (\%) \\
\hline Left inferior & $10(38.46)$ \\
Right inferior & $9(34.61)$ \\
Right superior & $3(11.53)$ \\
Left superior & 0 \\
Retrotracheal & $2(7.69)$ \\
Left superior Mediastinal & $1(3.84)$ \\
Failed to locate & $1(3.84)$ \\
\hline
\end{tabular}

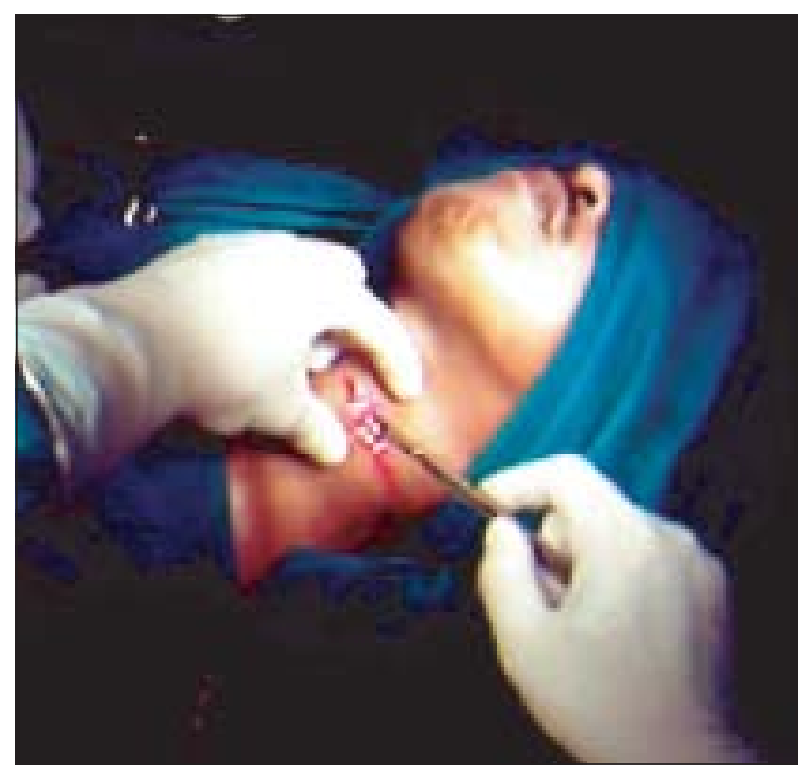

Fig.-1: Incision for 'focused parathyroidectomy' after local anaesthesia

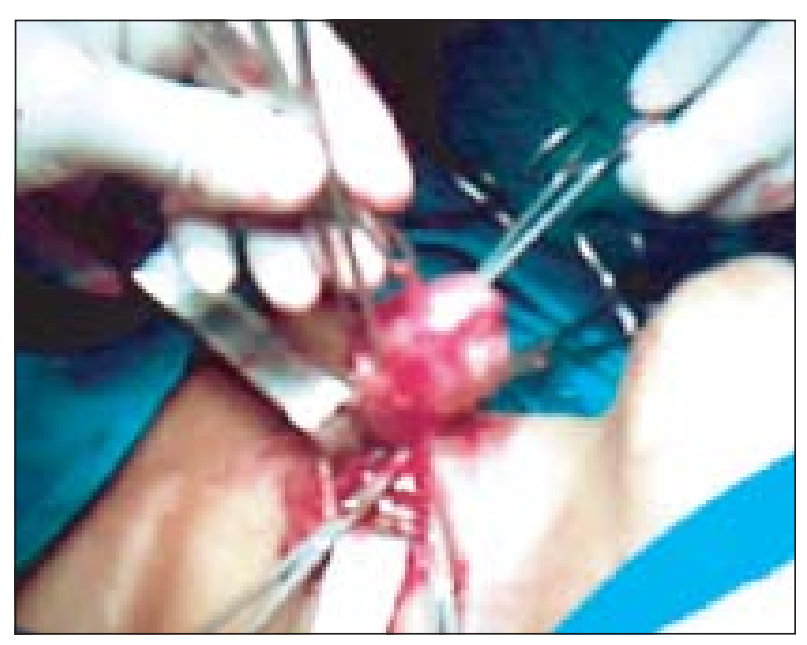

Fig.-2: Keyhole dissection and delivery of enlarged parathyroid

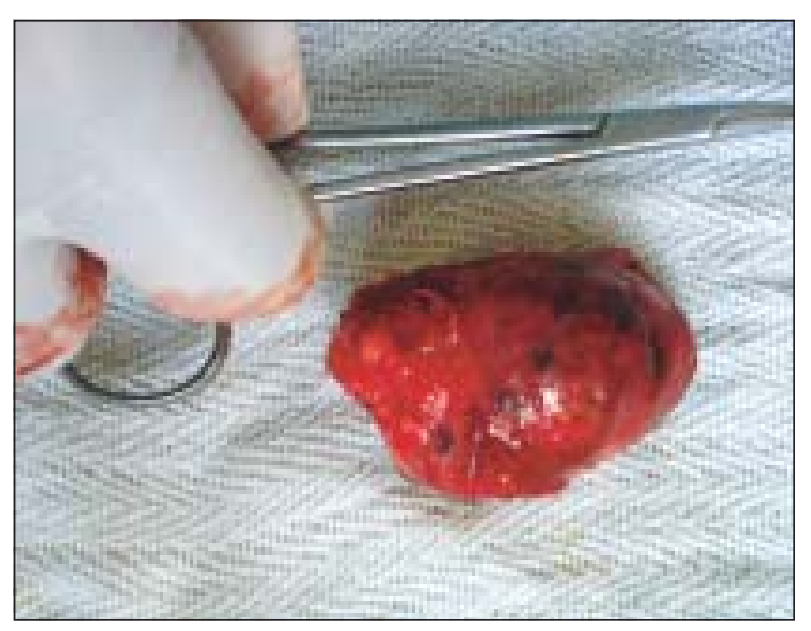

Fig. -3: Parathyroid gland after surgical removal 


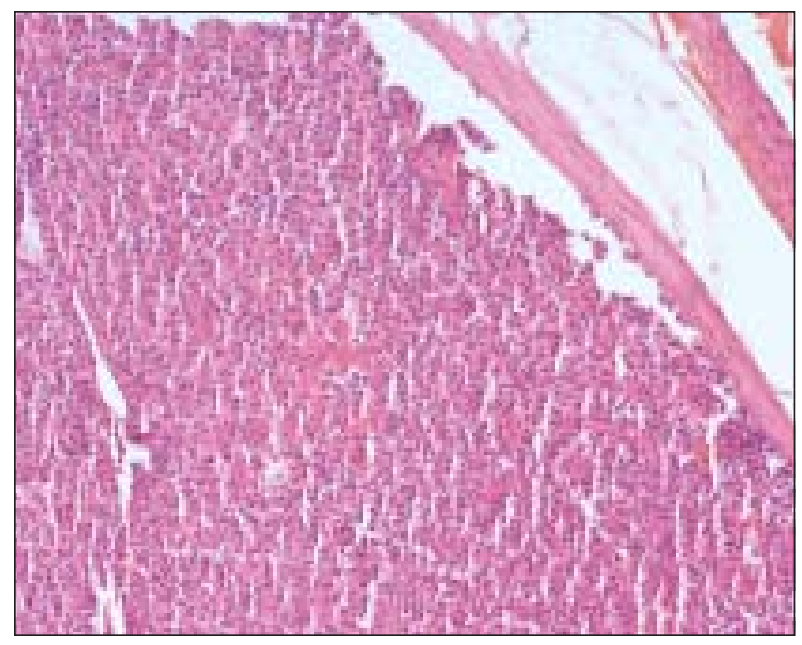

Fig.-4: Histopathology: Parathyroid adenoma in low magnification

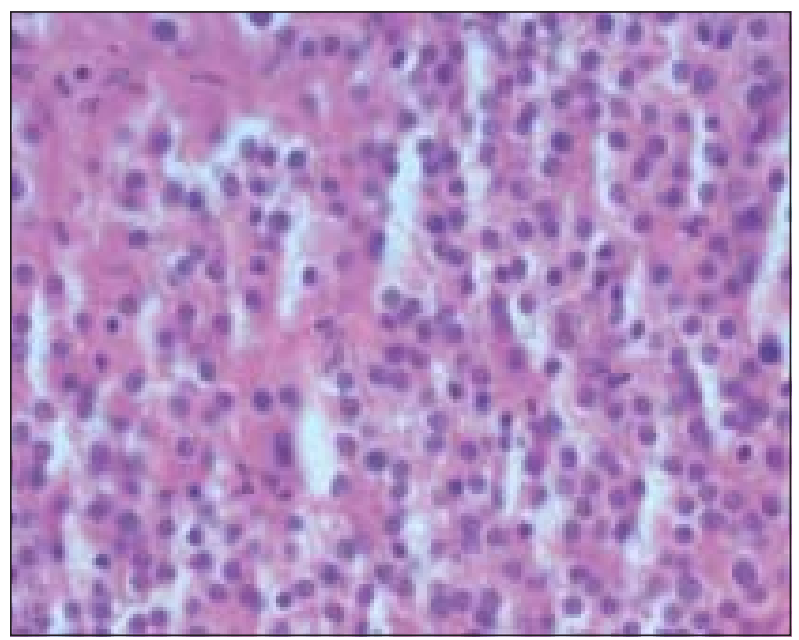

Fig. 5: Histopathology: Parathyroid adenoma in high magnification

Table-VII

Postoperative complications ( $n=26)$ :

\begin{tabular}{lccccc}
\hline Complication & BNE & UNE & Chi- sq & df & P value \\
& $\mathrm{N}=18$ & $\mathrm{~N}=8$ & value & & \\
\hline Hypocalcaemia & 15 & 7 & .074 & 1 & $1.00^{*}$ \\
Metabolic acidosis & 0 & 1 & 2.34 & 1 & $.308^{*}$ \\
Renal impairment & 1 & 1 & .376 & 1 & $.529^{*}$ \\
Cardiac dysrhythmia & 0 & 1 & 2.34 & 1 & $.308^{*}$ \\
Death & 0 & 2 & 4.87 & 1 & $.086^{*}$ \\
Recurrence after 1 year 1 & 0 & .462 & 1 & $1.00^{*}$ \\
\hline
\end{tabular}

* Fisher's Exact Test was done

\section{Discussion:}

Parathyroid gland controls blood level of calcium. Excessive parathormone in primary hyperparathyroidism (HPT) produces hypercalcaemia and if persists for prolonged period produces musculoskeletal, renal and cardioivascular compromise. So parathyroidectomy is advocated after diagnosis to reduce the risk of sequlae. It always needs specialized skill and experience with adequate information of anatomical variation and pathological appearance (reddish-brown).

The quasi-experimental study of 26 patients of HPT displayed some striking incidence in this perspective like $76.92 \%$ of patients being female within 31-50 years and overall M: F ratio 1:5.5. 57.69\% patients had blood intact PTH level more than 20 times upper normal i.e. >1000 $\mathrm{pg} / \mathrm{ml}$ and all the 26 patients had primary HPT due to parathyroid adenoma (100\%) which, to some extent, exceed global incidences. ${ }^{2}$ With the concept of multigland involvment removal of all glands $(3+1 / 2)$ by BNE was the principle in the past. But recent scintigraphic localization has changed the concept and proved the involvement of single gland in 85-90 ${ }^{1}$, which became the rationale for less traumatic but more precise 'focused' UNE pioneered by Roth et al ${ }^{7}$ and popularized by Tiblin $\mathrm{S}$ et al. ${ }^{14}$ Short incision, keyhole dissection and targeted ('focused') exploration to the gland to minimize operative time, hospital stay and postoperative complications are the principles. Different preoperative imaging localization was done in 22 patients with positive impression in 20(90.90\%). For sestamibi scan only 50\%(2/4) had positive impression which corresponds to the result of Ryan JA et al ${ }^{4}$ where he showed only $56 \%$ of abnormal gland in spite of multiglandular involvement but it didn't correspond to recent study of Slitt GT et al where he showed sensitivity of sestamibi scan as $70-80 \% 5,6$. Introducing use of new isotope in recent years may have any technological impact on result. During surgery, both the patients with negative scan had pathological glands. Local anaesthesia $(n=4)$ was used with similar success rate $(100 \%)$ as in general anaesthesia in well-localized glands. Inabett WB et al showed local anaesthesia as his approach of choice in asymptomatic primary HPT ${ }^{10}$. Balliski et al and Tiblin et al showed their success of surgery as $98.4 \%$, $96.2 \%$ and $98 \%{ }^{7,14}$ which correspond to our success rate i.e. $94.4 \%$ in BNE and $100 \%$ in focused UNE irrespective of type of anaesthesia. In this small number of patients with palpable glands there was no difference in significance testing $(\mathrm{p}=1.00)$. However, with time, corelation between impalpable gland-size and success of focused surgery under local anaesthesia needs to be 
ascertained on large series. The term 'success', here, defines surgical removal of enlarged gland and not in the sense of 'cure' as intra-operative biochemical frozen section was avoided due to lack of facility. However, avoidance of intra-operative PTH measurement was supported strongly by recent study of Mihai R et al. ${ }^{12}$ In superior mediastinal gland both cervical and video-guided thoracoscopic approach can be implemented. ${ }^{11}$ Here, 1 patient underwent extended UNE with successful gland removal. The overall failure rate of surgery was $3.84 \%$ ( $1 / 26$ ) which is comparable with the meta-analysis made by Balliski CR et al. ${ }^{7}$ Postoperative management needed attention to strict biochemical monitoring. The neglected patients responds very badly because persistent hypercalcaemia deranges cardiac and musculoskeletal contractility as well as renal function impairment due to loss of critical balance among PTH, ionic calcium, total body calcium and renal reabsorption of calcium and phosphate. After removal of excessive PTH producing adenoma most of the patients developed hypocalcaemia because PTH has a half-life of only 10-15 minutes and the suppressed glands cannot respond immediately by negative feedback mechanism. So, postoperative hypocalcaemia is almost inevitable in every case. Here, it was recorded in $84.61 \%(22 / 26)$ patients with recovery in $90.90 \%$ (20/22) after injectable calcium and subsequent oral calcium and vitamin D. Two neglected patients with very high PTH level had irreversible outcome due to severe musculoskeletal, renal and cardiovascular compromise. Non-availability of ICU enhanced deterioration and the overall mortality was $7.69 \%$ (2/26). To avoid the risk of renal and cardiovascular deterioration earlier removal of culpable gland should be done without delay after diagnosis and it is again strongly supported by recent study of Toft AD. ${ }^{13}$

\section{Conclusion:}

After diagnosis of hyperparathyroidism and preoperative localization unilateral 'focused parathyroidectomy' is a safe and effective option of gland removal. There is no difference in success of gland removal between 'focused' UNE and BNE $(\mathrm{p}=1.00)$. Use of local or general anaesthesia in well localized gland showed similar success (100\%) of surgery. Less operative time, hospital stay and postoperative morbidity and easier re-operation if recurs, are added advantages for the procedure. Surgical skill, experience and strict postoperative biochemical monitoring are essentials for good recovery. Delay in diagnosis and treatment are adverse factors. Surgery is recommended soon after diagnosis to reduce the risk of postoperative musculoskeletal, cardiac, renal and metabolic events.

\section{Acknowledgements:}

We are grateful to concerned professors of urology, nephrology, orthopaedics, endocrinology and cardiology for referring patients, attending to preoperative and postoperative consultation and giving follow-up information for accomplishment of the study. We also pay thanks to hospital authority and all ward and O.T. staff for co-operation and finally gratitude to all patients of this study.

\section{References:}

1. Johnson CD, Taylor I. Recent advances in surgery. $27^{\text {th }}$ ed. London. The Royal society of medicine press. 2004: 179-95.

2. Goldstein RE, Billheimer D, Martin WH, et al. Sestamibi scanning and minimally invasive radioguided parathyroidectomy without intraoperative parathyroid hormone measurement .Ann Surg 2003; 237: $722-31$

3. Bugis SP, Blliski CR. Unilateral exploration for primary hyperparathyroidism in the era of sestamibi scans. Can J Surg 2003; 46(suppl): 9.

4. Ryan JA, Eisenberg B, Pado KM, et al. Efficacy of selective unilateral exploration in hyperparathyroidism based on localization tests. Arch Surg 1997; 132: 886-91.

5. O’DohertyMJ, Kettle AG, Wells PC, et al. Parathyroid imaging with technetium 99m-sestamibi: Preoperative location and tissue uptake studies. J Nucl Med 1992; 33: 313-318.

6. Slitt G T, Lavery H, Morgan A, et al. Hyperparathyroidism but a negative sestamibi scan: a clinical dilemma. Am J Surg 2005; 190: 708-12.

7. Baliski CR, Stewart JK, Anderson DW, et al. Selective unilateral parathyroid exploration: an effective treatment for primary hyperparathyroidism. Am J Surg 2005; 189(5): 596-600.

8. Russell C. Unilateral neck exploration for primary hyperparathyroidism. Surg Clin North Am 2004; 84(3): 705-16.

9. Garnell EM, Thomas SK, McFarlane I, et al. Focused parathyroid surgery with intraoperative parathyroid hormone measurement, as an ambulatory procedure. Br J Sur 2004; 91: 78-82.

10. Inabnet WB, Fulla Y, Richard B, et al. Unilateral neck exploration under local anaesthesia: the approach of choice in asymptomatic primary hyperparathyroidism. Surgery 1999; 126: 1004-10.

11. Mekel M, Mahajana A, Ish-Shalom S, et al. Minimally invasive surgery for treatment of hyperparathyroidism. Isr Med Assoc J. 2005; 7(5): 323-27.

12. Mihai R, Palazzo FF, Gleeson FV, et al. Minimally invasive parathyroidectomy without parathyroid hormone monitoring in patients with primary hyperparathyroidism. Br J Surg 2007; 94: 4247.

13. Toft AD. Surgery for primary hyperparathyroidism - sooner rather than later. Lancet 2000; 355:1478-79.

14. Tiblin S, Bizard JP, Bondeson A, et al. Primary hyperparathyroidism due to solitary adenoma. Eur J Surg 1991; 157:511-15.

15. Sidhu S, Neil AK, Russel CF. Long term outcome of unilateral parathyroid exploration for primary hyperparathyroidism due to presumed parathyroid adenoma. World J Surg 2003; 27: 339-42. 